\section{Eventos adversos pós-vacinação em idosos no Estado de São Paulo, Brasil, de 2015 a 2017}

\author{
Post-vaccination adverse events in the elderly in \\ the State of São Paulo, Brazil, 2015 to 2017
}

Eventos adversos posvacunación en ancianos del
Estado de São Paulo, Brasil, de 2015 a 2017
Lais Cristina Barbosa dos Santos 1

Henrique Salmazo da Silva 2

Caroline Ribeiro Borja-Oliveira 1

Rosa Yuka Sato Chubaci 1

Beatriz Aparecida Ozello Gutierrez 1 doi: 10.1590/0102-311X00084820

\section{Resumo}

A vacinação é uma das ações de saúde pública que tem colaborado com a diminuição da incidência das doenças imunopreveníveis. No entanto, as vacinas podem acarretar eventos adversos pós-vacinação. Assim, este estudo teve como objetivos: analisar a prevalência dos eventos adversos pós-vacinação em pessoas idosas; levantar os eventos notificados; identificar as vacinas que causaram eventos e verificar os eventos adversos pós-vacinação e as vacinas administradas que acarretaram internações no Estado de São Paulo, Brasil, nos anos de 2015 a 2017. Estudo descritivo, transversal, de abordagem quantitativa com base nas notificações de eventos adversos pós-vacinação registradas no Sistema de Informações do Programa Nacional de Imunizações. Os resultados mostraram que dentre as 15.196 .080 pessoas idosas imunizadas, ocorreram 207 notificações de eventos adversos pós-vacinação, sendo 187 (89\%) devido a evento adverso não grave e 15 (8\%) por erro de imunização. A maioria dos acometidos era: do sexo feminino (86\%); raça branca (49\%); com idades de 60 a 69 anos (70\%). Dentre as manifestações clinicas destacamos as reações nos locais das aplicações (84\%). Constatou-se que 131 casos (64\%) evoluíram para cura sem sequelas. Em relação às internações, verificou-se que duas pessoas (2\%) foram hospitalizadas devido a efeito adverso grave, a primeira recebeu as vacinas: difteria/tétano adulto (dT), pneumocócica (Pn23) e influenza, e a segunda recebeu Pn23. Observaram-se informações incompletas nas notificações de eventos adversos pós-vacinação. Conclui-se que a notificação do eventos adversos pós-vacinação é essencial. Faz-se necessário o comprometimento dos profissionais no preenchimento adequado da notificação, e ainda, a supervisão da vigilância sanitária visando à qualidade da assistência prestada à pessoa idosa acometida por eventos adversos pós-vacinação.

Efeitos Colaterais e Reações Adversas Relacionados a Medicamentos;

Vacinação; Hospitalização; Idoso
Correspondência

B. A. O. Gutierrez

Escola de Artes, Ciências e Humanidades, Universidade de São Paulo.

Av. Arlindo Bétio 1000, São Paulo, SP 03828-000, Brasil. biagutierrez@yahoo.com.br

1 Escola de Artes, Ciências e Humanidades, Universidade de São Paulo, São Paulo, Brasil.

2 Universidade Católica de Brasília, Brasília, Brasil. 


\section{Introdução}

A vacinação é uma das ações de saúde pública que tem colaborado com a diminuição da incidência das doenças imunopreveníveis 1,2 .

No Brasil, a criação do Programa Nacional de Imunizações (PNI), em 1973, representou um progresso de grande relevância para o panorama da saúde pública. O PNI oferece para a população idosa em seu calendário nacional de vacinação e em Campanhas Nacionais de Vacinação cinco tipos de vacinas, sendo estas: difteria e tétano (dT), hepatite B, febre amarela, influenza e pneumocócica 23 (Pn23), disponibilizadas pelo Sistema Único de Saúde (SUS) 3,4.

Partindo da premissa de que as alterações imunológicas associadas ao envelhecimento tornam os idosos mais propensos às doenças, inclusive às preveníveis por vacinação, a oferta de programa de vacinação torna-se vital 5,6,7,8. Nessa abordagem, apesar dos programas de imunizações darem ênfase à população idosa, ainda não alcançaram os índices observados no Calendário de Vacinação da Criança. Com isso, os profissionais da saúde vêm tomando medidas de promoção e prevenção da saúde, orientando e estimulando a população idosa quanto à necessidade e importância do uso desse recurso de comprovado custo/benefício 8,9 .

Embora a eficácia das vacinas seja comprovada, os eventos adversos pós-vacinação são uns dos principais fatores que causam aversão nos idosos, resultando na possibilidade de não adesão à imunização $8,10,11,12,13$. Esse fato pode ser agravado porque na medida em que a cobertura vacinal aumenta e a doença diminui, aumenta o risco de eventos adversos pós-vacinação e, em consequência, diminui a adesão à vacinação, contribuindo para o ressurgimento das doenças já controladas 14,15.

Um estudo realizado em Campinas, São Paulo, com 1.517 idosos, mostrou que 36,7\% desta amostra acreditava que a vacina provoca reação, sendo este um dos motivos pelo qual os idosos não tomavam a vacina (influenza) 12. Num outro estudo, realizado no Município de Petrolina, Pernambuco, com 30 idosos, sendo 15 vacinados e 15 não vacinados contra a influenza, observou-se entre os não vacinados que mesmo conhecendo a finalidade da vacina (prevenir doenças) não aderiram à imunização devido aos seus eventos adversos 11 .

Os eventos adversos pós-vacinação são o surgimento de qualquer episódio clínico indesejado após a vacinação e que, nem sempre, está relacionado com a aplicação de vacina ${ }^{16}$. No entanto, existe a possibilidade do risco de eventos adversos pós-vacinação e a gravidade dos mesmos ser inferior ao das doenças contra as quais atuam ${ }^{14}$. Conforme a gravidade, os eventos adversos pós-vacinação são classificados em: evento adverso não grave, eventos não inclusos no evento adverso grave e evento adverso grave, sendo considerados graves aqueles que demandam internações por pelo menos $24 \mathrm{~h}$, causam sequela, anomalia congênita, risco de morte ou morte 16 .

Parte desses eventos pode se relacionar momentaneamente à utilização da vacina, mas não representar uma relação causal com a mesma, pois outras exposições ou causas atribuídas indevidamente às vacinas podem ser relatadas, o que exige a atuação da vigilância sanitária na notificação, estudo e investigação dos casos, especialmente quanto aos eventos graves e moderados 17 .

Entendendo a necessidade do monitoramento desses eventos, em 1998, foi inaugurado no Brasil o Sistema Passivo de Vigilância de Eventos Adversos Pós-vacinação, tendo como finalidade manter a segurança e a adesão à vacinação 1,18. Para que os eventos adversos não comprometam as ações do programa de imunização e cause redução das coberturas, bem como a insegurança epidemiológica de toda a população, são necessárias a notificação e a investigação de toda e qualquer ocorrência de eventos adversos pós-vacinação ${ }^{14}$. Salienta-se, ainda, a necessidade de identificar e analisar os eventos adversos pós-vacinação que mais acometem os idosos resultando em internações.

Diante desse contexto, o presente estudo tem como objetivo geral: analisar a prevalência dos eventos adversos pós-vacinação em pessoas idosas, e como objetivos específicos: levantar os eventos adversos pós-vacinação notificados; identificar as vacinas que causaram Eventos e suas consequências em idosos, e ainda levantar os dados não registrados na ficha de notificação dos eventos adversos pós-vacinação. 


\section{Métodos}

É um estudo descritivo, exploratório, transversal de abordagem quantitativa com base nas notificações de eventos adversos pós-vacinação registradas no Sistema de Informações do Programa Nacional de Imunizações (SI-PNI), módulo SI-EAPV. Os dados dessas notificações foram fornecidos pelo Centro de Vigilância Epidemiológica Prof. Alexandre Vranjac, localizado no Município de São Paulo, Brasil.

Para o desenvolvimento deste estudo foram utilizados dados secundários, relacionados à vacinação de pessoas com 60 anos ou mais, imunizadas no Estado de São Paulo, no período de 1o de janeiro de 2015 a 31 de dezembro de 2017.

Para os critérios de inclusão, foram adotados os eventos adversos pós-vacinação em pessoas idosas, com 60 anos ou mais de idade, das vacinas dT, hepatite B, influenza, Pn23 e a de febre amarela porque estas vacinas fazem parte da Caderneta de Vacinação do Idoso.

As variáveis analisadas foram aquelas existentes na ficha de notificação de eventos adversos pós-vacinação e erro de imunização: dados sociodemográficos (sexo, idade, raça); data de notificação; data de aplicação da vacina; tipo de imunobiológico administrado; dose, laboratório, lote, local de aplicação, indicação, especialidade; estabelecimento (Cadastro Nacional de Estabelecimentos de Saúde - CNES); manifestação; manifestação de evento adverso não grave, caracterizado como qualquer outro evento que não esteja nos critérios de evento adverso grave; ocorrência de evento adverso grave, situado como evento que requer hospitalização por pelo menos 24 horas ou prolongamento de hospitalização já existente, cursando com disfunção significativa e/ou incapacidade persistente (sequela); anomalia congênita; aumento do risco de morte (ou seja, induz a necessidade de uma intervenção clínica imediata para evitar o óbito); e óbito; documentação de erro de imunização e erro de imunização com evento adverso; evolução do caso; diagnóstico; atendimento médico; tipo de atendimento; registro de exames laboratoriais; tempo de hospitalização.

Foram excluídas as notificações duplicadas.

Um banco de dados foi construído com o auxílio do software Epi Info versão 7.2 (https://www. cdc.gov/epiinfo/index.html) e analisado por meio do software Excel 2010 (https://products.office. $\mathrm{com} /$ ). A amostra foi caracterizada com a distribuição de frequências absolutas e relativas de todas as variáveis analisadas e os resultados estão apresentados em tabelas.

O projeto de pesquisa foi submetido e aprovado pelo Comitê de Ética em Pesquisa da Escola de Artes, Ciências e Humanidades, Universidade de São Paulo (CEP-EACH-USP) por meio do protocolo: 2.945.678. Foram respeitados os preceitos da Resolução no 466/2012 do Conselho Nacional de Saúde, relacionados à ética em pesquisa com seres humanos.

\section{Resultados}

No Estado de São Paulo, no período de 2015 a 2017, foram imunizadas 15.196 .080 pessoas com 60 anos ou mais 19 (SI-PNI. http://sipni-gestao.datasus.gov.br/si-pni-web/faces/relatorio/consolidado/ dosesAplicadasCampanhaInfluenzaFaixa.jsf, acessado em/03/Jun/2020). Desse total, foram encontradas 207 notificações de eventos adversos pós-vacinação.

Os resultados relacionados aos dados sociodemográficos da Tabela 1 mostram que, do total de 207 notificações analisadas, a maioria da amostra era do sexo feminino (86\%), de idades entre 60 e 69 (70\%), e de raça branca (49\%). A Tabela 1 mostra ainda que dessas notificações realizadas foram identificados 187 (89\%) registros por evento adverso não grave. Observou-se que erro de imunização com evento adverso ocorreu apenas em um caso.

Segundo a Tabela 2, das 187 notificações por evento adverso não grave, na maioria dos casos o indivíduo recebeu mais de uma vacina no mesmo dia. Dentre as vacinas estudadas, a dT aparece em 115 (56\%) das notificações.

Esclarecemos que das cinco notificações de evento adverso grave presentes na Tabela 2, os idosos receberam mais de um imunobiológico. No entanto, a vacina da hepatite B não foi aplicada em nenhum dos idosos que apresentaram evento adverso grave. 
Tabela 1

Características da amostra e tipo de evento pós-vacinação. Estado de São Paulo, Brasil, de 2015-2017.

\begin{tabular}{|c|c|c|c|c|}
\hline Variáveis & $\begin{array}{l}2015 \\
\text { n (\%) }\end{array}$ & $\begin{array}{l}2016 \\
\text { n (\%) }\end{array}$ & $\begin{array}{l}2017 \\
\text { n (\%) }\end{array}$ & $\begin{array}{l}\text { Total } \\
\text { n (\%) }\end{array}$ \\
\hline \multicolumn{5}{|l|}{ Sexo } \\
\hline Feminino & $14(78)$ & $64(82)$ & $101(91)$ & $179(86)$ \\
\hline Masculino & $4(22)$ & $14(18)$ & $10(9)$ & $28(14)$ \\
\hline Total & $18(100)$ & $78(100)$ & $111(100)$ & $207(100)$ \\
\hline \multicolumn{5}{|l|}{ Idade (anos) } \\
\hline $60-69$ & - & - & - & $145(70)$ \\
\hline $70-79$ & - & - & - & $53(26)$ \\
\hline $80+$ & - & - & - & $9(4)$ \\
\hline Total & - & - & - & $207(100)$ \\
\hline Mediana & - & - & - & 65 \\
\hline \multicolumn{5}{|l|}{ Raça } \\
\hline Branca & $10(56)$ & $37(47)$ & $55(50)$ & $102(49)$ \\
\hline Amarela & $1(5)$ & $2(3)$ & $8(8)$ & $11(5)$ \\
\hline Negra & $1(5)$ & $1(1)$ & $0(0)$ & $2(1)$ \\
\hline Parda & $1(5)$ & $7(9)$ & $7(7)$ & $15(7)$ \\
\hline Informações não preenchidas & $5(29)$ & $31(40)$ & $41(41)$ & $77(38)$ \\
\hline Total & $18(100)$ & $78(100)$ & $111(111)$ & $207(100)$ \\
\hline \multicolumn{5}{|l|}{ Tipo de evento } \\
\hline Evento adverso grave & $2(11)$ & $2(2)$ & $1(1)$ & $5(3)$ \\
\hline Evento adevrso não grave & $16(89)$ & $70(90)$ & $101(91)$ & $187(89)$ \\
\hline Erro de imunização & $0(0)$ & $6(8)$ & $8(7)$ & $14(7)$ \\
\hline Erro de imunização com evento adverso & $0(0)$ & $0(0)$ & $1(1)$ & $1(1)$ \\
\hline Total & $18(100)$ & $78(100)$ & $111(100)$ & $207(100)$ \\
\hline
\end{tabular}

Fonte: Sistema de Informações do Programa Nacional de Imunizações (SI-PNI), módulo SI-EAPV.

\section{Tabela 2}

Tipos de vacina e eventos adversos pós-vacinação em idosos. Estado de São Paulo, Brasil, 2015-2017.

\begin{tabular}{|c|c|c|c|c|c|}
\hline Vacina & $\begin{array}{c}\text { Erro de } \\
\text { imunização }\end{array}$ & $\begin{array}{l}\text { Erro de imunização } \\
\text { com evento adverso }\end{array}$ & $\begin{array}{c}\text { Erro de } \\
\text { imunização grave }\end{array}$ & $\begin{array}{l}\text { Erro de imunização } \\
\text { não grave }\end{array}$ & Total \\
\hline Pn23 & & 1 & 1 & 58 & 65 \\
\hline $\operatorname{Pn} 23, \mathrm{dT}$ & & & & 2 & 2 \\
\hline Pn23, dT, hepatite $B$, influenza & & & & 1 & 1 \\
\hline Pn23, influenza & & & 1 & 5 & 6 \\
\hline dT & 4 & & & 100 & 104 \\
\hline dT, Pn23, influenza & & & 2 & & 2 \\
\hline dT, influenza & & & 1 & 3 & 4 \\
\hline dT, heptatite B & & & & 8 & 8 \\
\hline dT, hepatite B, influenza & & & & 1 & 1 \\
\hline Heaptite B & 2 & & & 8 & 10 \\
\hline Hepatite B, influenza & & & & 1 & 1 \\
\hline Total & 14 & 1 & 5 & 187 & 207 \\
\hline
\end{tabular}

dT: difteria e tétano; Pn23: pneumocócica 233.

Fonte: Sistema de Informações do Programa Nacional de Imunizações (SI-PNI), módulo SI-EAPV. 
De acordo com a Tabela 3, as manifestações mais frequentes nos eventos adversos pós-vacinação foram as reações locais (84\%). Se um ou mais desses sinais e sintomas forem considerados uma única síndrome de reação local, esta síndrome corresponderia a 576 de todos os eventos adversos notificados.

Ao analisar a assistência prestada aos idosos que apresentaram eventos adversos, verificou-se que 102 (49\%) tiveram atendimento médico e apenas dois idosos foram internados conforme dados apresentados na Tabela 4.

\section{Tabela 3}

Tipos de manifestações pós-vacinação. Estado de São Paulo, Brasil, 2015-2017.

\begin{tabular}{lcc}
\hline Variáveis & $\mathbf{n}$ & $\%$ \\
\hline Reações locais & 576 & 84 \\
Náuseas e vômitos & 13 & 2 \\
Febre $\geq 39^{\circ} \mathrm{C}$ (axilar) & 13 & 2 \\
Cefaleia & 13 & 2 \\
Celulite & 11 & 2 \\
Exantema & 9 & 1 \\
Hematoma & 7 & 1 \\
Sonolência & 4 & 1 \\
Febre $\leq 39^{\circ} \mathrm{C}$ (axilar) & 3 & 1 \\
Outros & 37 & 4 \\
Total & 686 & 100 \\
\hline
\end{tabular}

Fonte: Sistema de Informações do Programa Nacional de Imunizações (SI-PNI), módulo SI-EAPV.

\section{Tabela 4}

Atendimento médico, tipos de atendimentos, exames laboratoriais pós-vacinação e evolução do caso. Estado de São Paulo, Brasil, 2015-2017.

\begin{tabular}{|c|c|c|}
\hline Variáveis & $\mathbf{n}$ & $\%$ \\
\hline \multicolumn{3}{|l|}{ Atendimento médico } \\
\hline $\operatorname{Sim}$ & 102 & 49 \\
\hline Não & 50 & 24 \\
\hline Ignorado & 55 & 27 \\
\hline Total & 207 & 100 \\
\hline \multicolumn{3}{|l|}{ Tipos de atendimentos } \\
\hline Consulta médica & 81 & 79 \\
\hline Internação & 2 & 2 \\
\hline Informações não preenchidas & 19 & 19 \\
\hline Total & 102 & 100 \\
\hline \multicolumn{3}{|l|}{ Exames laboratoriais } \\
\hline Sim & 3 & 1 \\
\hline Não & 99 & 48 \\
\hline Informações não preenchidas & 105 & 51 \\
\hline Total & 207 & 100 \\
\hline \multicolumn{3}{|l|}{ Evolução do caso } \\
\hline Cura sem sequela & 131 & 64 \\
\hline Cura com sequela & 7 & 3 \\
\hline Em acompanhamento & 29 & 14 \\
\hline Informações não preenchidas & 40 & 19 \\
\hline Total & 207 & 100 \\
\hline
\end{tabular}

Fonte: Sistema de Informações do Programa Nacional de Imunizações (SI-PNI), módulo SI-EAPV. 
Com base na Tabela 5, na qual são apresentadas as informações não preenchidas pós-vacinação, é possível visualizar que o registro de exames laboratoriais se destacou, totalizando $51 \%$ de ausência de registro.

Baseando-se nos resultados do banco de dados institucional do SI-PNI/SI-EAPV, coletados durante o mês de dezembro de 2018, identificou-se que a primeira internação após eventos adversos pós-vacinação foi de idoso com 60 anos, do sexo masculino, raça não informada e que recebeu os imunobiológicos dT, Pn23 e influenza. Esse evento foi notificado como evento adverso grave com manifestação clínica sistêmica: presença de parestesia, sem identificação do local de ocorrência e diagnóstico de síndrome de Guillain-Barré. O tempo de internação foi de 14 dias com evolução do caso em acompanhamento. Já a outra internação foi de idosa com 77 anos, do sexo feminino, raça branca e que recebeu a vacina Pn23, notificada como evento adverso grave apresentando manifestações locais: calor, celulite, dor, edema, eritema ou rubor e artralgia com o diagnóstico: celulite no local da administração da vacina e o tempo de permanência hospitalar foi de três dias. A paciente evoluiu para cura sem sequelas.

Constatou-se que das 207 notificações foram identificados 15 (7\%) registros decorrentes de erro de imunização/erro de imunização com evento adverso, sendo 14 (93\%) para erro de imunização e 1 (7\%) para erro de imunização com evento adverso. Salienta-se que o caso de erro de imunização com eventos adversos ocorreu em uma idosa com 92 anos, de cor amarela, na qual foi administrada a vacina Pn23, caso de revacinação.

Salienta-se que no banco de dados do SI-PNI/SI-EAPV disponibilizado para este estudo não havia registro de eventos relacionados com a vacina da febre amarela devido a problemas no sistema de processamento de dados.

\section{Discussão}

O presente artigo analisou os eventos adversos pós-vacinação em idosos no Estado de São Paulo de 2015 a 2017. Este estudo vai ao encontro da demanda do Centro de Vigilância Epidemiológica Prof. Alexandre Vranjac, que desde 2015 assumiu a nova sistemática da vigilância de eventos adversos pós-vacinação contando com: nova ficha de notificação e um novo sistema de informação intitulado de SI-PNI/SI-EAPV. A partir de então, o Estado de São Paulo iniciou treinamentos nas suas diversas regiões ao longo dos anos de 2015, 2016 e 2017 visando à capacitação dos profissionais da área de saúde quanto à farmacovigilância de vacinas relacionadas ao processo de detecção, avaliação, compreensão, prevenção e comunicação de eventos adversos pós-vacinação ou qualquer outro problema relacionado à vacina ou à imunização, o que resultou em um aumento progressivo de todas as notificações de eventos adversos pós-vacinação 17. Esse aumento pode ser visualizado nos resultados

\section{Tabela 5}

Informações não preenchidas nas 207 fichas de notificação de eventos adversos pós-vacinação. Estado de São Paulo, Brasil, 2015-2017.

\begin{tabular}{|c|c|c|c|c|}
\hline Variáveis & $\begin{array}{l}2015 \\
\text { n (\%) }\end{array}$ & $\begin{array}{l}2016 \\
\text { n (\%) }\end{array}$ & $\begin{array}{l}2017 \\
\text { n (\%) }\end{array}$ & $\begin{array}{l}\text { Total } \\
\text { n (\%) }\end{array}$ \\
\hline Raça & $5(2,4)$ & $26(12,6)$ & $24(11,6)$ & $55(26,6)$ \\
\hline Laboratório farmacêutico & $2(1,0)$ & $5(2,4)$ & $6(2,9)$ & $13(6,3)$ \\
\hline Via de administração & $0(0,0)$ & $3(1,4)$ & $3(1,4)$ & $6(2,9)$ \\
\hline Local de administração & $0(0,0)$ & $3(1,4)$ & $2(1,0)$ & $5(2,4)$ \\
\hline Atendimento médico & $5(2,4)$ & $19(9,2)$ & $31(14,8)$ & $55(26,6)$ \\
\hline Registro de exames laboratoriais & $17(8,2)$ & $76(36,7)$ & $111(53,6)$ & $204(98,6)$ \\
\hline Evolução do caso & $1(0,5)$ & $15(7,2)$ & $24(11,6)$ & $40(19,3)$ \\
\hline
\end{tabular}

Fonte: Sistema de Informações do Programa Nacional de Imunizações (SI-PNI), módulo SI-EAPV. 
apresentados neste estudo, porém, ainda com falhas no preenchimento das variáveis da ficha de notificação que foram deixadas em branco.

Em relação às variáveis sociodemográficas, o estudo constatou que os eventos adversos pós-vacinação foram verificados em maior quantidade nas mulheres do que nos homens, resultado que corrobora outros trabalhos 6,12,20,21,22,23. Esse resultado pode estar relacionado à maior presença das mulheres na população idosa, pois a superioridade desta população aumenta com a idade e a maior longevidade delas em comparação aos homens 6,24. Outro motivo que também pode estar atrelado é a maior busca das mulheres por atendimento nos serviços de saúde, cuidando mais da saúde e aderindo melhor às campanhas de prevenção 6,8,22.

Também, como constatado em outros estudos, a faixa etária mais atingida foi a de 60 a 69 anos $6,12,21,22$. Tal fato pode estar relacionado ao acesso mais facilitado dessa população aos serviços de saúde por ser mais autônoma e independente do que a população de idosos mais longevos, situados em idades mais avançadas e com maior presença de limitações funcionais e cognitivas 6 .

No que se refere à raça, a maioria foi classificada como branca, também corroborando os achados em outras pesquisas 12,20,22. No entanto, um dos estudos concluiu que não houve associação entre vacinação e raça 12 .

Os resultados encontrados neste trabalho mostraram que as notificações por eventos adversos não graves são as mais frequentes, corroborando os achados em outras pesquisas 22,25 . Isso reforça a segurança das vacinas aplicadas. No entanto, corrobora-se que a subnotificação desses eventos dificulta a sua generalização 25 .

Ainda em relação aos eventos adversos não graves, considera-se importante a realização de avaliação clínica e exames laboratoriais para a elucidação do diagnóstico etiológico e causal, visando a esclarecimentos sobre o ocorrido e não simplesmente relacionar os eventos adversos pós-vacinação à vacina, assim será possível o encerramento adequado do caso suspeito 25 .

Dentre os tipos de eventos adversos pós-vacinação que apresentaram maior frequência estão as reações locais, como por exemplo, dor, eritema ou rubor, edema e calor, estando de acordo com os verificados em outras pesquisas 6,22,26,27. Essas reações locais podem ser resultado do ato de introduzir a agulha e, com isto, causar lesão muscular e irritação no local e/ou reação provocada pelo imunobiológico administrado. Nesse contexto, outro fator a se considerar também são as outras substâncias utilizadas nas vacinas, como o adjuvante de hidróxido de alumínio, com potencial de ocasionar uma reação inflamatória no local da aplicação 6,16.

Cabe destacar que a imunização é realizada, principalmente, pela equipe de enfermagem, e o erro de imunização com evento adverso pode acontecer se as normas técnicas não forem cumpridas. Nesse sentido, um estudo mostrou que a taxa de crescimento para esse erro, no período estudado de 2003 a 2013, foi de 0,18, apontando um crescimento anual, e ainda foi estimado um valor médio para o período de 2014 a 2018, com variação de 2,54 em 2014 a 3,27 em 2018, apontando tendência crescente para esses erros 1 .

O presente trabalho se propôs a verificar se algum evento adverso pós-vacinação notificado foi motivo de internação hospitalar e a resposta encontrada foi positiva em dois casos. Apesar da síndrome de Guillain-Barré ter sido diagnosticada em um dos casos, foi impossível especificar o nome da vacina que possa ter causado este evento no idoso porque houve administração concomitante de três vacinas: dT, Pn23 e influenza.

Sabe-se que a síndrome de Guillain-Barré é uma manifestação neurológica a que o próprio vírus da influenza pode estar associado 28 . No entanto, sua frequência é menor do que um caso por milhão de doses aplicadas, sendo esse risco menor que as complicações da influenza prevenidas pela vacina ${ }^{29}$. Entretanto, é desconhecido se a vacina da influenza pode aumentar o risco da síndrome de Guillain-Barré, ademais, estudos que buscaram esta relação mostraram resultados contraditórios 16.

A população idosa quando comparada aos adultos jovens, é mais propensa a adquirir doenças infecciosas, aumentando assim o número de morbidade, mortalidade e hospitalizações ${ }^{9}$. Um estudo desenvolvido no Estado do Paraná, com dados referentes às internações ocorridas no período de 1995 a 2005, demonstrou que as doenças do aparelho respiratório ocuparam a segunda causa de internação, e que após a intervenção vacinal contra influenza este índice foi significativamente menor, passando de 23,7 em 1995 para 18,06 em 200530. 
Outro trabalho investigou a morbidade relatada por idosos vacinados e não vacinados contra a influenza em Porto Alegre, Rio Grande do Sul. Esses achados indicaram que o número de internações hospitalares foi mais frequente nos não vacinados, com 26 internações contra nove internações para os vacinados 31 .

No segundo caso de internação devido aos eventos adversos pós-vacinação foi utilizada apenas a vacina Pn23. O diagnóstico descrito foi de celulite no sítio de administração. Em outro estudo observou-se reações locais intensas onde a vacina foi administrada, como dor, edema e eritema ou rubor relacionados principalmente à Pn23 6. Contrapondo a esses resultados, um estudo realizado com 152 pessoas com idade média de 61,8 anos encontrou que 23,8\% delas apresentaram reações no local da aplicação da vacina, sendo considerada pouco reatogênica na primeira dose e com um único caso em que os sintomas persistiram por mais de seis dias 32 .

Sabe-se que os idosos e as crianças são as mais suscetíveis de serem colonizados e invadidos pelo Streptococcus pneumoniae, causando doenças graves: pneumonia bacteriana, sepse e meningite ${ }^{33}$. Ressalta-se que devido às limitações das vacinas pneumocócicas relacionadas à proteção de curta duração, cobertura limitada de sorotipos e o fator idade como agravante decorrente do envelhecimento biológico, é relevante a administração da Pn23 nos idosos (Merck Vaccines. Pneumovax 23: indication for Pneumovax 23. https://www.merckvaccines.com/pneumovax23, acessado em 08/Jul/2020).

Quanto à ocorrência do erro de imunização com evento adverso, um trabalho que analisou 604 registros de eventos adversos pós-vacinação relacionado a erro de imunização no período de 2003 a 2013, no Estado do Paraná, encontrou que a notificação que registrou maior percentual (57\%) de evento adverso foi relacionada à vacina BCG, presente no Calendário de Vacinação da Criança, e na distribuição das faixas etárias, as crianças menores de um ano foram as mais atingidas com (70,5\%) 1. Nesse mesmo trabalho, o percentual de eventos adversos pós-vacinação decorrentes de erro de imunização na faixa etária 60 anos e mais foi de $1 \%$ dos 604 registros. Os autores relataram que a ocorrência de eventos adversos pós-vacinação por erro de imunização vem aumentando, podendo ser reflexo da melhoria da vigilância em notificar ou deficiência na prática da enfermagem nas vacinas 1 .

Ainda sobre o erro de imunização com evento adverso, outro estudo clínico realizado com indivíduos a partir de 65 anos ou mais revelou que as reações adversas sistêmicas encontradas relacionadas à Pn23 foram maiores após a revacinação, ao ser comparada à vacinação inicial (Merck Vaccines. Pneumovax 23: indication for Pneumovax 23. https://www.merckvaccines.com/pneumovax23, acessado em 08/Jul/2020). A falta de dados relacionada à notificação do erro de imunização com evento adverso, no atual estudo, inviabiliza a análise da situação.

Nesse contexto, ressalta-se a importância da conscientização de todos os profissionais de saúde que atuam na área de imunização de que na presença de evento adverso é essencial questionar o idoso para se certificar se é revacinação e quantas vezes esta pessoa já foi vacinada com este imunobiológico. Dessa forma, os profissionais do Centro de Vigilância Epidemiológica Prof. Alexandre Vranjac poderão atuar de maneira efetiva no que tange ao levantamento dos problemas causadores do evento e, ainda, na proposta de soluções adequadas no sentido de trazer segurança à vítima do evento, ao profissional que administrou a vacina, à equipe de saúde que está atendendo ao idoso e também à sociedade em geral que faz uso dessa vacina.

Uma pesquisa realizada objetivando levantar a incidência de eventos adversos pós-vacinação em crianças encontrou que a vacina pentavalente, que previne a difteria, tétano, coqueluche, meningite por Haemophilus tipo b e poliomielite foi a segunda a apresentar o maior número de notificações 34 . Também encontrou que, na maioria das vezes, as crianças tinham recebido simultaneamente mais de um tipo de vacina ${ }^{34}$, associação permitida pelo Ministério da Saúde 35.

A elevada prevalência de eventos adversos pós-vacinação relacionados à vacina dT pode estar relacionada às reações já esperadas, como dor, vermelhidão e febre nas primeiras 72 horas após a vacinação 35. Contudo, um estudo europeu conduzido com 2.100 adultos da Áustria, Bélgica, Alemanha, Grécia, Itália e Polônia observou que com o avanço da idade as concentrações de anticorpos específicos para tétano e difteria foram menores, especialmente em países com níveis geralmente baixos de anticorpos, como Itália, Polônia e Grécia 36. Houve variabilidade na porcentagem de pessoas com concentrações de anticorpos abaixo do nível de proteção $(0,1 \mathrm{UI} / \mathrm{mL})$, que variaram de $2 \%$ a $31 \%$ no tétano e de $28 \%$ a $63 \%$ na difteria 36 . Curiosamente, as concentrações de anticorpos específicos para o 
tétano foram mais elevadas nos homens do que nas mulheres, o que provavelmente se deve à vacinação durante o serviço militar ou a vacinas de reforço mais frequentes após lesões 36 .

Outro estudo que comparou um grupo de idosos e de adultos jovens com relação ao nível de proteção para difteria e tétano na janela de cinco anos, observou ao final que $24 \%$ dos adultos jovens e 54\% do grupo de idosos estavam desprotegidos contra a difteria. As respostas das células T ao tétano e à difteria não diferiram entre jovens e idosos, mas devido à imunosenescência e/ou vacinação insuficiente mais cedo na vida; os idosos podem ser considerados um grupo de risco para a difteria 37. Dessa forma, torna-se necessária a orientação e a insistência para que os idosos recebam a vacina dT.

A notificação contribui para a supervisão da qualidade da vacinação e dos imunobiológicos, buscando, assim, identificar as possíveis causas para se ter uma melhor segurança e qualidade na assistência. Nesse sentido, o aumento das notificações apresentado em 2017 pode não estar, necessariamente, relacionado ao aumento dos eventos, mas sim à maior adesão ao sistema decorrente dos benefícios ocorridos com as mudanças do sistema SI-PNI/SI-EAPV e da realização de programas de capacitação dos profissionais de saúde em relação à notificação dos eventos adversos. No entanto, ainda persistiram informações não preenchidas na ficha de notificação.

Diante dos achados deste estudo, considera-se fundamental utilizar-se de algumas estratégias para a conscientização do idoso sobre a importância da prevenção de doenças por meio da vacinação. Assim, a atenção interprofissional no sentido de esclarecer o idoso sobre a vacinação, no sentido de alertá-lo quanto aos riscos e aos benefícios; prestar atendimento domiciliário; implementar campanhas de vacinas específicas para os idosos; capacitar um membro da equipe do serviço de saúde para realizar o recrutamento dos idosos no período de vacinação; realizar divulgação e orientação sobre as vacinas sistematicamente 8 .

Nessa linha de pensamento, determinar a melhor estratégia e quais as ferramentas mais assertivas a serem usadas nas campanhas de vacinação visando à proteção dos idosos ainda precisam ser desbravadas.

Apesar dos dados apresentados serem um retrato dos eventos adversos pós-vacinação na população idosa do Estado de São Paulo no período de 2015 a 2017, algumas limitações podem ser destacadas como a possível subnotificação de caso: a ausência de dados completos a respeito da qualidade das doses aplicadas; ausência de informações sobre o tipo de atendimento que o paciente recebeu e nos tipos de manifestações apresentadas, gerando comprometimento da compreensão da real situação dos casos. Adicionalmente, detectou-se falta da especificação da vacina causadora do eventos adversos pós-vacinação, dificultando a análise dos dados quando estes eram realizados simultaneamente a outras vacinas, e não foi possível avaliar os dados sobre a febre amarela porque eles não estavam consolidados para análise, e por isto não foram disponibilizados para o nosso estudo.

\section{Conclusão}

Observou-se, por meio dos achados deste trabalho, que a chance do idoso ter um evento adversos grave pós-vacinação é pequena. Nesse contexto, esses resultados podem favorecer na divulgação das campanhas de vacinação, pois trazem subsídios aos profissionais para que possam esclarecer e incentivar a população idosa quanto aos riscos das vacinas, além de desmistificar a existência de possíveis preconceitos em relação a certos tipos de vacinas.

Já para os profissionais de saúde, este estudo traz à tona o alerta quanto à necessidade de observarem na história pregressa da pessoa idosa a existência de imunização recente. Também, esses resultados mostram a necessidade de melhor preenchimento da ficha de notificação dos eventos adversos pós-vacinação, de forma a melhorar as ações de vigilância e controle das reações adversas. Assim, destaca-se a relevância da notificação completa, pois esses dados facilitarão tanto o tratamento clínico do paciente quanto a veracidade do estado de saúde da população idosa brasileira pós-vacinação.

Enfim, são necessárias tanto a capacitação sistematizada dos profissionais de saúde em relação à vacinação quanto à supervisão da notificação dos eventos adversos pós-vacinação por parte dos centros de vigilância sanitária em São Paulo e no Brasil. O fortalecimento das ações de imunização para pessoa idosa torna-se vital para a prevenção de causas evitáveis de morte por doenças infectocontagiosas, o que torna essas ações um dos alvos importantes da saúde pública brasileira. 


\section{Colaboradores}

L. C. B. Santos contribuiu na revisão da literatura, coleta de dados, análise dos resultados e revisão da versão final do artigo. H. S. Silva auxiliou na revisão crítica relevante do conteúdo intelectual da versão final do artigo. C. R. Borja-Oliveira contribuiu na análise dos dados e finalização do texto do artigo. R. Y. S. Chubaci participou na análise dos resultados e na revisão do texto do artigo. B. A. O. Gutierrez colaborou na revisão da literatura, metodologia, análise dos resultados e revisão da versão final do artigo.

\section{Informações adicionais}

ORCID: Lais Cristina Barbosa dos Santos (00000002-6540-3658); Henrique Salmazo da Silva (0000-0002-3888-4214); Caroline Ribeiro Borja-Oliveira (0000-0003-3735-4016); Rosa Yuka Sato Chubaci (0000-0001-9993-0889); Beatriz Aparecida Ozello Gutierrez (0000-0001-6901-6439).

\section{Agradecimentos}

Agradecemos ao Centro de Vigilância Epidemiológica Prof. Alexandre Vranjac e a Eder Gatti Fernandes por ter colaborado na coleta e análise dos dados.

\section{Referências}

1. Bisetto LHL, Ciosak SI. Análise da ocorrência de evento adverso pós-vacinação decorrente de erro de imunização. Rev Bras Enferm 2017; 70:81-9.

2. Barreto ML, Bastos FI, Ximenes RAA, Barata $\mathrm{RB}$, Rodrigues LCMD. Successes and failures in the control of infectious diseases in Brazil: social and environmental context, policies, interventions, and research needs. Lancet 2011; 377:1877-89.

3. Moraes LRM, Piantola MAF, Pereira SA, CastroI JT, Santos FAOII, Ferreira LCS. Eventos adversos de vacinas e as consequências da não vacinação: uma análise crítica. Rev Saúde Pública 2018; 52:40.

4. Ministério da Saúde. Ministério recomenda: é preciso envelhecer com saúde. http:// portalms.saude.gov.br/noticias/agencia-sau de/25924-ministerio-recomenda-e-preci so-envelhecer-com-saude (acessado em 21/ Mai/2018).

5. Veiga AMV. Imunidade e envelhecimento. In: Freitas EV, Py L, organizadoras. Tratado de geriatria e gerontologia. 3a Ed. Rio de Janeiro: Guanabara Koogan; 2013. p. 894-903.

6. Linheira-Bisetto LH, Ciosak SI, Cordeiro TLR, Boing MS. Ocorrência de eventos adversos pós-vacina em idosos. Cogitare Enferm 2016; 21:1-10.

7. Haq K, Mc Elhaney J. Immunosenescence: influenza vaccination and the elderly. Curr Opin Immunol 2014; 29:38-42.

8. Santos EP, Oliveira MM. Desafios da vacinação. Imunizações 2018; 11:10-5.

9. Toniolo Neto J, Rodrigo F, Kairalla MC. Vacinas. In: Freitas EV, Py L, organizadoras. Tratado de geriatria e gerontologia. 3a Ed. Rio de Janeiro: Guanabara Koogan; 2013. p. 904-16.

10. Ballalai I, Bravo F. Imunização: tudo o que você sempre quis saber. Rio de Janeiro: Rmcom; 2016.

11. Silva SPC, Menandro MCS. Representações de idosos sobre a vacina da gripe. Ciênc Saúde Colet 2013; 18:2179-88.

12. Francisco PMSB, Barros MBA, Cordeiro MRD. Vacinação contra influenza em idosos: prevalência, fatores associados e motivos da não-adesão em Campinas, São Paulo, Brasil. Cad Saúde Pública 2011; 27:417-26.

13. Moura RF, Andrade FB, Duarte YAO, Lebrão ML, Antunes JLF. Fatores associados à adesão à vacinação anti-influenza em idosos não institucionalizados, São Paulo, Brasil. Cad Saúde Pública 2015; 31:2157-68.

14. Sato APS, Ferreira VLR, Taui MC, Rodrigues LC, Barros MB, Martineli E, et al. Uso de registro informatizado de imunização na vigilância de eventos adversos pós-vacina. Rev Saúde Pública 2018; 52:4.

15. Chen R, Rastogi SC, Mullen JR, Hayes SW, Cochi SL, Donlon JA, et al. The vaccine adverse event reporting system (VAERS). Vaccine 1994; 12:42-50. 
16. Departamento de Vigilância das Doenças Transmissíveis, Secretaria de Vigilância em Saúde, Ministério da Saúde. Manual de vigilância epidemiológica de eventos adversos pós-vacinação. 3a Ed. Brasília: Ministério da Saúde; 2014.

17. Bisetto LHL, Cubas MR, Malucelli A. A prática da enfermagem frente aos eventos adversos pós-vacinação. Rev Esc Enferm USP 2011; 45:1128-34

18. Monteiro SAMG, Takano AO, Waldman EA. Avaliação do sistema brasileiro de vigilância de eventos adversos pós-vacinação. Rev Bras Epidemiol 2011; 14:361-71.

19. Centro de Vigilância Epidemiológica "Prof. Alexandre Vranjac”. Imunização. http://sau de.sp.gov.br/cve-centro-de-vigilancia-epi demiologica-prof.-alexandre-vranjac/areasde-vigilancia/imunizacao/ (acessado em 22/ Mar/2020).

20. Campos EC, Sudan LCP, Mattos ED, Fidelis R. Fatores relacionados à vacinação contra a gripe em idosos: estudo transversal, Cambé, Paraná, Brasil. Cad Saúde Pública 2012; 28:878-88.

21. Santos BRL. Situação vacinal e associação com a qualidade de vida, a funcionalidade e a motivação para o autocuidado em idosos. Rev Bras Epidemiol 2009; 12:533-40.

22. Moura RF, Andrade FB, Duarte YAO, Lebrão ML, Antunes JLF. Fatores associados à adesão à vacinação anti-influenza em idosos não institucionalizados, São Paulo, Brasil. Cad Saúde Pública 2015; 31:2157-68.

23. Tambara DR, Mallmann DG, Santos NO, Pereira FW, Hammerschmidt KSA, Girondi JBR. Perfil multidimensional dos idosos participantes da campanha de vacinação contra influenza. Rev Bras Geriatr Gerontol 2015; 18:84554.

24. Instituto Brasileiro de Geografia e Estatística. Pesquisa Nacional por Amostra de Domicílios: síntese de indicadores 2013. 2a Ed. Rio de Janeiro: Instituto Brasileiro de Geografia e Estatística; 2015.

25. Pacheco FC, Domingues CMS, Maranhão AGK, Carvalho SMD, Teixeira AMS, Braz RM, et al. Análise do Sistema de Informação da Vigilância de Eventos Adversos Pós-Vacinação no Brasil, 2014 a 2016. Rev Panam Salud Pública 2018; 42:e12.

26. Piacentini S, Contrera-Moreno L. Eventos adversos pós-vacinais no Município de Campo Grande (MS, Brasil). Ciênc Saúde Colet 2011; 16:531-6.
27. Rodrigues D, Dalri RCB. Eventos adversos pós-vacinação contra influenza em idosos no Brasil. Rev Salud Pública 2019; 21:22-8.

28. Aires YRFF, Lima ALS, Sampaio PGG, Galdino GS. Síndrome de Guillain-Barré associada à imunização H1N1. Rev Bras Neurol Psiquiatr 2014; 18:73-6.

29. Ferrer ALM, Marcon SS, Santana RG. Hospital morbidity among elderly patients, before and after influenza vaccination in the state of Paraná. Rev Latinoam Enferm 2008; 16:83237.

30. Vilarino MAM, Lopes MJM, Bueno ALM, Brito MRV. Idosos vacinados e não vacinados contra a influenza: morbidade relatada e aspectos sociodemográficos. Ciênc Saúde Colet 2010; 15:2879-86.

31. Centers for Disease Control and Prevention. Preliminary results: surveillance for GuillainBarre syndrome after receipt of influenza A (H1N1) 2009 monovalent vaccine - United States, 2009-2010. MMWR Morb Mortal Wkly Rep 2010; 59:657-61.

32. Donalisio MR, Rodrigues SMCP, Mendes ET, Krutman M. Eventos adversos após vacinação contra o pneumococo. J Bras Pneumol 2007; 33:51-6.

33. van de Garde MDB, Knol MJ, Rots NY, van Baarle D, van Els CACM. Vaccines to protect older adults against pneumococcal disease. Interdiscip Top Gerontol Geriatr 2020; 43:11330.

34. Braga PCV, Silva AEBC, Mochizuki LB, Lima JC, Sousa MRG, Bezerra ALQ. Incidência de eventos adversos pós-vacinais em crianças. Rev Enferm UFPE 2017; 11:4126-35.

35. Ministério da Saúde. Manual de normas e procedimentos de vacinação. Brasília: Ministério da Saúde; 2014.

36. Weinberger B, Keller M, Breitenberger CPD, Koller B, Fiegl S, Moreno-Villanueva M, et al. Protection against tetanus and diphtheria in Europe: the impact of age, gender and country of origin based on data from the MARK-AGE Study. Exp Gerontol 2017; 105:109-12.

37. Grasse M, Meryk A, Schirmer M, GrubeckLoebenstein B, Weinberger B. Booster vaccination against tetanus and diphtheria: insufficient protection against diphtheria in young and elderly adults. Immun Ageing 2016; 13:26. 


\section{Abstract}

Vaccination is one of the public health measures that has most contributed to decreasing the incidence of vaccine-preventable diseases. However, vaccines can lead to post-vaccination adverse events. This study thus aimed to analyze the prevalence of post-vaccination adverse events in elderly persons, determine the reported post-vaccination adverse events, identify the vaccines that cause post-vaccination adverse events, and verify the post-vaccination adverse events and vaccines that lead to hospitalizations in the State of São Paulo, Brazil, from 2015 to 2017. This was a descriptive cross-sectional study with a quantitative approach based on notifications of post-vaccination adverse events recorded in the Information System of the National Immunization Program. The results showed that of the 15,196,080 elderly persons that were immunized, there were $207 \mathrm{re}$ ports of post-vaccination adverse events, of which 187 (89\%) were non-serious adverse events and 15 (8\%) due to immunization errors (IE). The majority of the patients were female (86\%), white (49\%), and from 60 to 69 years of age (70\%). Clinical manifestations featured local reactions at the injection site (84\%). There were 131 cases (64\%) that evolved to cure without sequelae. Two individuals (2\%) were hospitalized due to serious adverse events. The first had received adult diphtheria/ tetanus (dT), pneumococcal (Pn23), and influenza vaccines, and the second had received $P n 23$. The post-vaccination adverse events notifications showed incomplete information. In conclusion, notification of post-vaccination adverse events is essential. Health professionals need to be committed to adequate completion of the notification forms, with health surveillance supervision aimed at quality of care for elderly persons with postvaccination adverse events.

Drug-Related Side Effects and Adverse Reactions; Vaccination; Hospitalization; Aged

\section{Resumen}

La vacunación es una de las acciones de salud pública que ha colaborado a la disminución de la incidencia de enfermedades inmunoprevenibles. No obstante, las vacunas pueden acarrear eventos adversos de posvacunación. Por ello, este estudio tuvo como objetivos: analizar la prevalencia de los eventos adversos de posvacunación en personas ancianas; recoger los eventos adversos de posvacunación notificados; identificar las vacunas que causaron eventos eventos adversos de posvacunación y verificar los eventos eventos adversos de posvacunación y las vacunas administradas que provocaron internamientos en el Estado de São Paulo, Brasil, durante los años de 2015 a 2017. Estudio descriptivo, transversal, de abordaje cuantitativo basado en las notificaciones de eventos adversos de posvacunación, registradas en el Sistema de Información del Programa Nacional de Inmunizaciones. Los resultados mostraron que, entre las 15.196.080 personas ancianas inmunizadas, ocurrieron 207 notificaciones de eventos adversos de posvacunación, siendo 187 (89\%) debidas a un evento adverso no grave y 15 (8\%) por error de inmunización. La mayoría de los afectados era del sexo femenino (86\%); raza blanca (49\%); con edad de 60 a 69 años (70\%). Entre las manifestaciones clínicas se destacaron las reacciones en los lugares de la aplicación de la vacuna (84\%). Se constató que 131 casos (64\%) evolucionaron a una cura sin secuelas. En relación con los internamientos se verificó que dos personas (2\%) fueron hospitalizadas, debido a un efecto adverso grave, la primera recibió las vacunas: difteria/tétano adulto (dT), neumocócica (Pn23) y gripe y la segunda recibió Pn23. Se observó información incompleta en las notificaciones de eventos adversos de posvacunación. Se concluye que la notificación del eventos adversos de posvacunación es esencial. Es necesario el compromiso de los profesionales en la cumplimentación adecuada de la notificación e incluso, la supervisión de la vigilancia sanitaria, con el fin de analizar la calidad de la asistencia prestada a la persona anciana afectada por eventos adversos pós- vacinação.

Efectos Colaterales y Reacciones Adversas Relacionados con Medicamentos; Vacunación; Hospitalización; Anciano
Recebido em 17/Abr/2020

Versão final reapresentada em 29/Jul/2020

Aprovado em 10/Ago/2020 